\title{
Prediction of Dilute Phase Pneumatic Conveying Characteristics using MP-PIC Method
}

\author{
K. Amila Chandra W.K. Hiromi Ariyaratne Morten C. Melaaen \\ Faculty of Technology, Natural Sciences and Maritime Sciences - University College of Southeast Norway, Post box 235, \\ N-3603 Kongsberg, Norway, \{amila.c.kahawalage, hiromi.ariyaratne, \\ morten.c.melaaen\} dusn. no
}

\begin{abstract}
Pneumatic conveying characteristics of a dilute phase flow in a circular horizontal pipe was predicted using MP-PIC method in OpenFOAM code. The geometry, material and operating conditions are similar to some experimental data in published literature. The pipe diameter is $30.5 \mathrm{~mm}$. The solid particles are plastic pellets which are having $1000 \mathrm{~kg} / \mathrm{m}^{3}$ of density and 0.2 $\mathrm{mm}$ of particle diameter. The simulations were carried out for $10 \mathrm{~m} / \mathrm{s}$ of superficial air velocity and for different solids mass loadings $0,1,2$ and 3 . The pressure drop, air velocity profiles and solids distribution were analysed and some of the results were compared with experimental data from the literature. The predicted pressure drops and air velocity profiles show a quite good agreement with the experimental data.
\end{abstract}

Keywords: MP-PIC, OpenFOAM, pneumatic conveying, simulations, experimental data

\section{Introduction}

Pneumatic conveying systems are employed to transfer powders, granules and other dry bulk materials through pipes or tubes. The main attractive features of the pneumatic conveying systems are; the flexibility, completely enclosed system and having less moving parts compared to the other mechanical transport systems. One of the principal disadvantages of these systems is the requirement of higher horsepower, because the blower or compressor does the primary work. For better system performance and optimal energy usage, the selected blower or compressor characteristics should be matched with the system characteristics. In that scenario, the air flow rate and the pressure drop through the system are the major key factors when choosing a suitable blower or compressor.

There are two different ways of pneumatic conveying; as dilute phase and dense phase. In the dilute phase conveying, particles are fully suspended in the conveying air. On the other hand in dense phase, the particles are conveyed as fluidized dunes or as discrete plugs of material without much suspension of the material. The remarkable differences in the operational condition for the different modes are; the velocity and the pressure. In dilute phase conveying, relatively a high velocity and a low pressure are employed. Due to high operating velocity in dilute phase, the system requires excessive power. Moreover, operational problems may arise such as particle attrition and erosive wear of the pipelines. The pressure drops for pneumatic conveying systems have widely been measured experimentally by many researchers for different pipe configurations, particle sizes and solids loading ratios (Hyder et al., 2000; Mason and Li, 2000; Tsuji and Morikawa, 1982).

In last few decades, computational fluid dynamics (CFD) is intensively used in modeling, designing and optimizing of pneumatic transport systems. Quite many commercial and open source software programmes are available for that purpose (Bilirgen and Levy, 2001; Chu and $\mathrm{Yu}, 2008$; Hidayat and Rasmuson, 2005; Huber and Sommerfeld, 1998; Laín and Sommerfeld, 2008; Lee et al., 2004; Levy and Mason, 1998; Mason and Levy, 1998).

In general, commercial CFD softwares are user friendly with respect to many aspects such as mesh generation, solution algorithms and visualization. Nevertheless, the modification of source code of those software packages according to user requirement is not very straight forward. Moreover, the costs of commercial licenses are also significant. OpenFOAM is an open source CFD simulation software package and can be used in wide variety of flow simulation applications. It is a finite volume solver. The CFD code can be developed according to the user requirements, as example for a certain specific application. And the code is also for free of charge. Due to the above reasons, OpenFOAM is popular in both academic and industrial sector.

Currently, multiphase particle-in-cell (MP-PIC) method is widely employed in solving gas-solids flow systems. This is also referred as computational particle fluid dynamics (CPFD) in some literature. This is an Euler-Lagrange approach which treats the particles in a discrete manner. Particles are treated as parcels in MPPIC method and each parcel consists of a definite 
number of real particles of the same properties such as size, density, temperature, etc. The method has been quite much used and verified for certain applications such as bubbling and circulating fluidized beds, fluidized bed gasifiers, fluidized beds for carbon capture and gas/liquid/solids fluidized beds (Chen et al., 2013; Karimipour and Pugsley, 2012; Liang et al., 2014; Parker et al., 2013). However, published information about use of this method in predicting pneumatic conveying characteristics is not found.

The solid phase normal stress is used to compute the particle-particle interactions near the close pack limit, but not directly through modeling of particle collisions (Snider, 2001). Besides, the particle collisions are not considered implicitly in MP-PIC method, hence time step size can be increased. Due to that, particle and flow calculation can be computed using same time step size and it reduces the computational time for the simulation. All these benefits make MP-PIC method more suitable for the simulation of the large-scale particulate flow systems. However, in dilute systems the instantaneous and binary contacts are more significant compared to enduring contacts which are modeled through normal stress model. In addition to solid phase normal stress, binary and instantaneous collisions are modeled through new terms developed by Snider and O'Rourke (O'Rourke and Snider, 2012; O'Rourke and Snider, 2010).

In the present study, some of the experimental data found in the literature are reproduced (Tsuji and Morikawa, 1982). Three dimensional simulations are carried out using MP-PIC method in OpenFOAM code. A horizontal circular pipe conveying plastic pellets in dilute phase is simulated. Pressure drop, air velocity profiles and solids distribution are analyzed and some of those results are compared with the experimental data.

\section{Model Formulation and Methodology}

\subsection{Mathematical Model}

The mass and momentum equations are solved for the gas phase. For the solid phase, Liouville equation is solved for the distribution function which is a function of particle positions, velocities and sizes (Andrews and O'Rourke, 1996; Snider, 2001). In the equations, refers to the gradient respect to the direction and refers to the gradient respect to the velocity. The mass and momentum equation for the gas phase are shown in (1) and (2), respectively.

$$
\begin{gathered}
\frac{\partial\left(\varepsilon \rho_{g}\right)}{\partial t}+\nabla_{x}\left(\varepsilon \rho_{g} u_{g}\right)=0 \\
\frac{\partial\left(\varepsilon \rho_{g} u_{g}\right)}{\partial t}+\nabla_{x}\left(\varepsilon \rho_{g} u_{g} u_{g}\right)+\nabla_{x} p=-F+\varepsilon \rho_{g} g+\nabla \varepsilon \tau_{g}
\end{gathered}
$$

The gas phase stress tensor is given by,

$$
\tau_{g}=\mu_{e f f}\left(\nabla u_{g}+\nabla u_{g}^{T}\right)-\frac{2}{3} \mu_{e f f} \nabla \cdot u_{g} I
$$

Where $\varepsilon, \rho_{g}, u_{g}, p, g \tau_{g}, \mu_{\text {eff }}, I$ are the gas volume fraction (or void fraction), the gas density, the gas velocity vector, the gas pressure, the accerlation due to gravity, the gas stress tensor, effective viscosity and unit tensor, respectively. The turbulent viscosity is solved using the modified k-epsilon equation for multiphase flows (not presented here). The rate of momentum exchange per unit volume from the gas to the particle phase is denoted by (13). The gas pressure and density are correlated by (4).

$$
\frac{p}{\rho_{g}^{\gamma}}=\text { constant }
$$

The particle phase is described by Liouville equation (5). where $f(x, v, m, t)$ is called the particle distribution function and $x, v, m$ and $t$ represent the particle position, the particle velocity, the particle mass and the time, respectively. More detail about collision term (on the right hand side of (6)) can be found in elsewhere (O'Rourke and Snider, 2012; O'Rourke and Snider, 2010; Snider, 2001).

$$
\begin{gathered}
\frac{\partial f}{\partial t}+\nabla_{x}(f v)+\nabla_{v}(f A)=\left(\frac{\partial f}{\partial t}\right)_{\text {coll }} \\
\left(\frac{\partial f}{\partial t}\right)_{\text {coll }}=\frac{f_{D}-f}{\tau_{D}}+\frac{f_{G}-f}{\tau_{G}}
\end{gathered}
$$

The particle velocity is given by,

$$
\frac{d x}{d t}=v
$$

$A=d v / d t$ is the particle acceleration which is given by

$$
A=D\left(u_{g}-v\right)-\frac{1}{\rho_{s}} \nabla_{x} p+g-\frac{1}{\theta \rho_{s}} \nabla_{x} \tau
$$

where $D, \rho_{s}$ and $\tau$ are the drag function, the particle density and the isotropic solids stress, respectively. Drag function is given by (9).

$$
D=C_{d} \frac{3}{8} \frac{\rho_{g}}{\rho_{s}} \frac{\left|u_{g}-v\right|}{R}
$$

Where $C_{d}$ is drag coefficient which is modeled from Wen-Yu drag model (Shah et al., 2015) and $\mathrm{R}$ is particle radius. Expression for the isotropic solids stress has been taken from (Harris and Crighton, 1994) and shown in (10). 


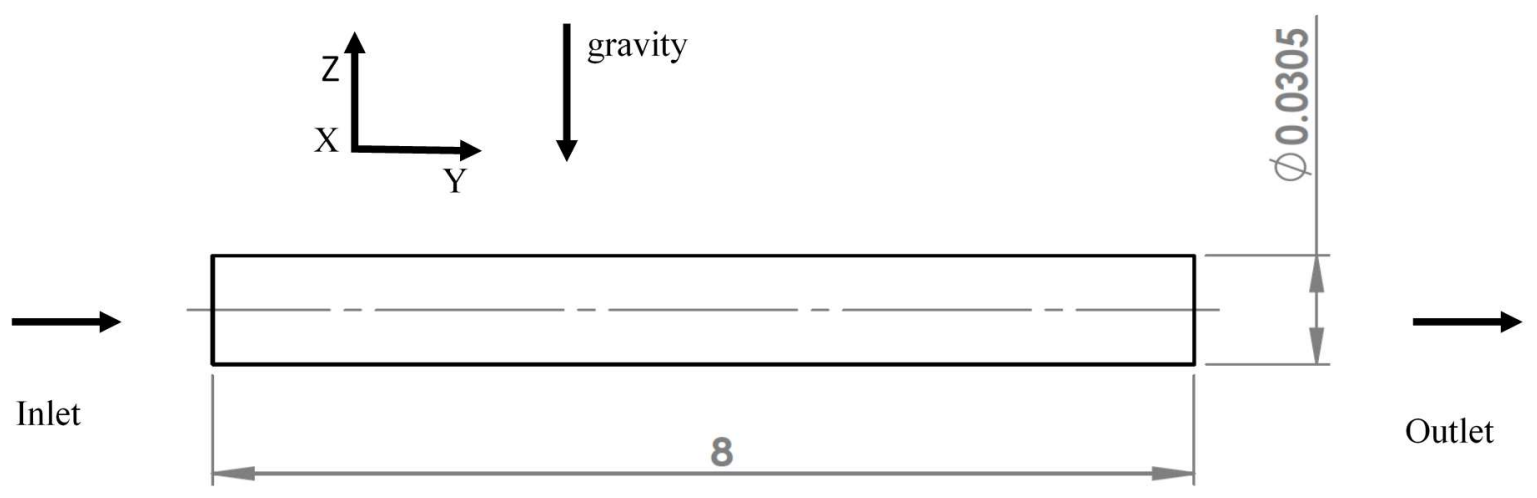

Figure 1. Sketch of the geometry of the computational domain (units are in meters).

$$
\tau=P_{s} \frac{\theta^{\beta}}{\max \left[\theta_{c p}-\theta, \omega(1-\theta)\right]}
$$

In (10), $P_{s}$ is a constant with units of pressure and $\theta_{c p}$ is the particle-phase volume fraction at close pack, respectively. $\beta$ is a constant $(2 \leq \beta \leq 5)$ and $\omega$ is a small number in the order of 10-7. The particle volume fraction is related to the distribution function by,

$$
\theta=\iint f \frac{m}{\rho_{s}} d m d v
$$

Then $\varepsilon$ and $\theta$ are related by,

$$
\theta+\varepsilon=1
$$

To complete the equation, we need an expression for the interphase momentum transfer function $F$ and it is defined as (13).

$$
F=\iint f m\left[D\left(u_{G}-v\right)-\frac{1}{\rho_{s}} \nabla p\right] d m d v
$$

\subsection{Experiment and Simulation Procedure}

Tsuji and Morikawa (1982) have conducted experiments for gas-solids two phase flow in a horizontal pipe. The pipe diameter is $30.5 \mathrm{~mm}$. The pressure drops and also particle and air velocities have been measured using laser-Doppler velocimeter (LDV). Plastic pellets which are having particle density of 1000 $\mathrm{kg} / \mathrm{m}^{3}$ have been used as the solid material.

They have conducted experiments for two different mean particle sizes; $0.2 \mathrm{~mm}$ and $3.6 \mathrm{~mm}$ by varying superficial air velocity and solids mass loading. The velocities range from 6 to $20 \mathrm{~m} / \mathrm{s}$ and the solids mass loadings range from 0 to 6 . However in the present study, the simulations are conducted for mean particle size of $0.2 \mathrm{~mm}$. The used air density and viscosity are $1.225 \mathrm{~kg} / \mathrm{m}^{3}$ and $1.46073 \times 10^{-5} \mathrm{~Pa} \mathrm{~s}$, respectively. The simulations are carried out for four different mass loadings; $0,1,2$ and 3 . The superficial air velocity for each case is $10 \mathrm{~m} / \mathrm{s}$. The Reynolds number of the flow is around 21000. Description of each simulation case is shown in Table 1.

Table 1. Simulation case description.

\begin{tabular}{|l|l|l|l|l|}
\hline Case & $\begin{array}{l}\text { Solids to } \\
\text { air mass } \\
\text { flow } \\
\text { ratio }\end{array}$ & $\begin{array}{l}\text { Air } \\
\text { mass } \\
\text { flow } \\
\text { rate } \\
(\mathrm{kg} / \mathrm{s})\end{array}$ & $\begin{array}{l}\text { Solids } \\
\text { mass } \\
\text { flow rate } \\
(\mathrm{kg} / \mathrm{s})\end{array}$ & $\begin{array}{l}\text { Particle } \\
\text { volume } \\
\text { fraction } \\
\text { at inlet } \\
(\%)\end{array}$ \\
\hline Case_0 & 0 & 0.009 & 0.000 & 0.00 \\
\hline Case_1 & 1 & 0.009 & 0.009 & 0.12 \\
\hline Case_2 & 2 & 0.009 & 0.018 & 0.24 \\
\hline Case_3 & 3 & 0.009 & 0.027 & 0.32 \\
\hline
\end{tabular}

\subsection{Geometry and Meshing}

The computational domain is an $8 \mathrm{~m}$ long horizontal pipe having $30.5 \mathrm{~mm}$ of diameter. This is shown in Figure 1. Three-dimensional geometry was generated and meshed using SALOME 7.5.1. To obtain better accuracy and convergence, hexahedral type elements were selected. The grid is uniform and consists of 126000 elements (Figure 2).

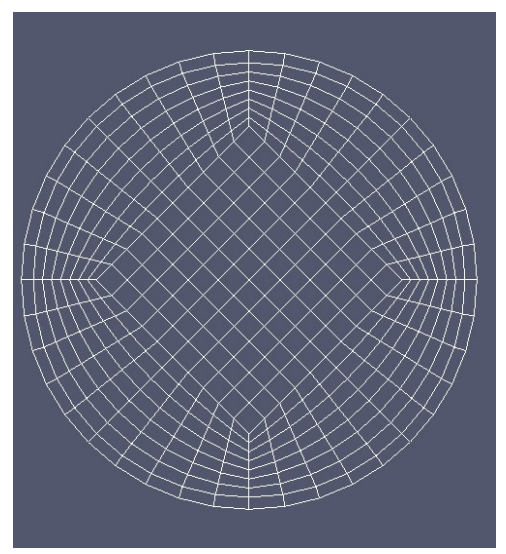

Figure 2. Mesh of pipe cross section. 


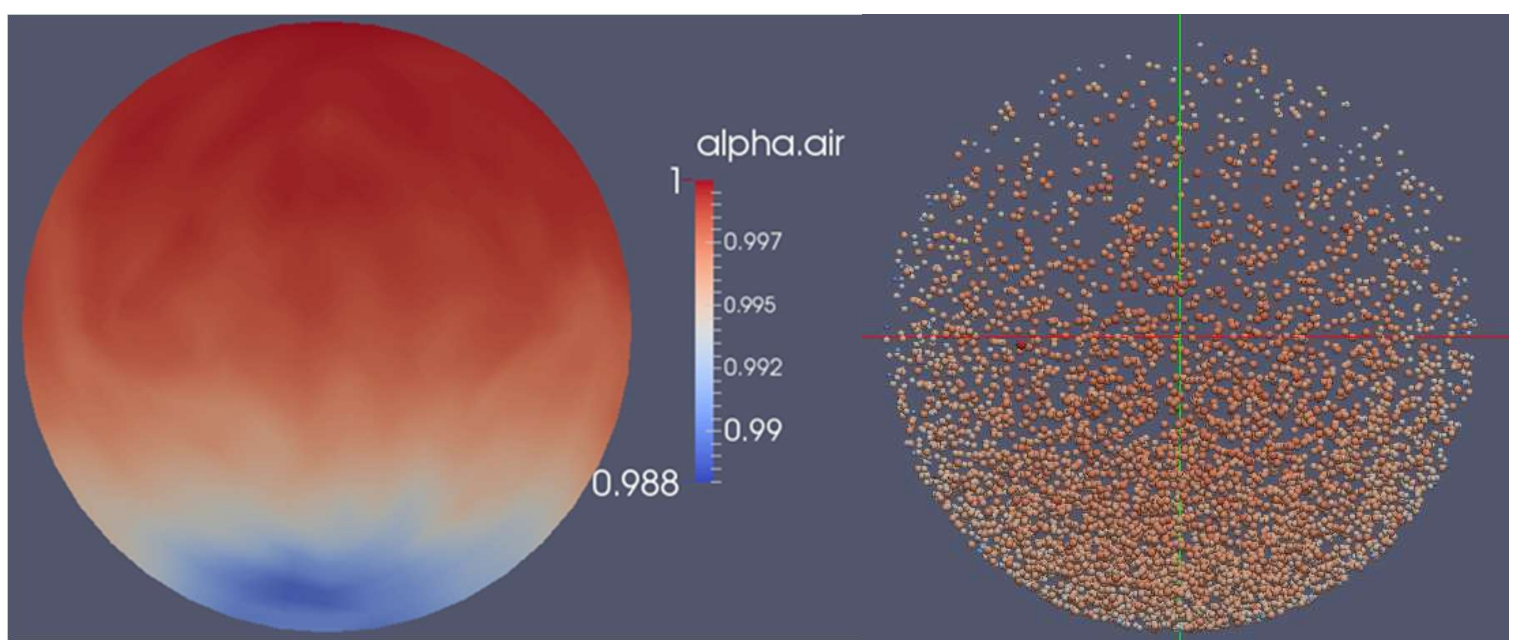

(a)

(b)

Figure 3. (a) Air volume fraction and (b) Particle distribution at outlet after $1.8 \mathrm{~s}$ for the Case_ 2 .

\subsection{Boundary Conditions and Solution} Method

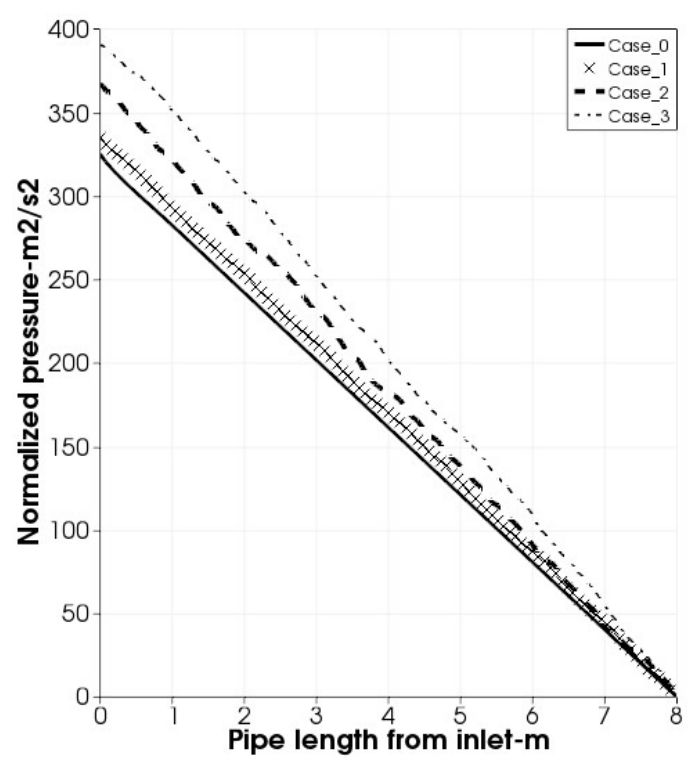

Figure 4. Normalized pressure along the pipe centre line at $1.8 \mathrm{~s}$.

As shown in Figure 1, the system consists of three boundaries as the inlet, the outlet and the wall. Pressure at the outlet was defined as zero gauge pressure and superficial air velocity at the inlet was defined as $10 \mathrm{~m} / \mathrm{s}$. Particle-particle interactions nearby close pack limit are modeled using particle normal stress model.

The isotropic solids stress was defined as (10) and $P_{s}$ $, \theta_{c p}, \beta$ and $\omega$ are specified as $1,0.6,3$ and $10^{-8}$, respectively. Particle to wall interaction was modeled with restitution coefficients and its value is 0.95 . Collisional return-to-isotropy of particle velocity fields which are important for dilute systems (O'Rourke and Snider, 2012) are modeled from time scale model.

In that model, particle-phase volume fraction at close pack and particle-particle restitution coefficient are specified as 0.6 and 0.95 , respectively. MPPICFoam was used as the solver in OpenFOAM. Simulation was run in transient mode at time step size of $0.0001 \mathrm{~s}$ until it comes to quasi-steady state which was confirmed by monitoring the pressure at certain points.

\section{Results and Discussion}

The pressure drop in a pneumatic conveying system is a very crucial property because it will affect to the performance of the blower or the compressor. Pressure drops along the horizontal pipe center line at $1.8 \mathrm{~s}$ (normalized by the air density) for four cases are shown in Figure 4. Case_0 which corresponds to only air flow shows quite stable pressure fall along the pipe length; however the pressure drop profiles start to fluctuate with the solids loadings. Moreover, the total pressure drop increases with the increase of solids loadings, which is physically reasonable for any dilute system. When the solids loading increases for a certain size of particles, the particle number density in the system increases accordingly. Higher number of particles in the system causes high frequent particle-particle and particle-wall collisions. This enhances the particle energy dissipation resulting in high drag force and also increased pressure drop.

Table 2 shows the comparison of experimental data (Tsuji and Morikawa, 1982) with predicted quasi-steady time-averaged pressure drops in fully developed region along the pipe centre line for different cases. The simulation results show quite good agreement with the experimental data. However, it seems that the error is increased with an increase of solids loadings. It should be noted that the pressure drops are simulated with 


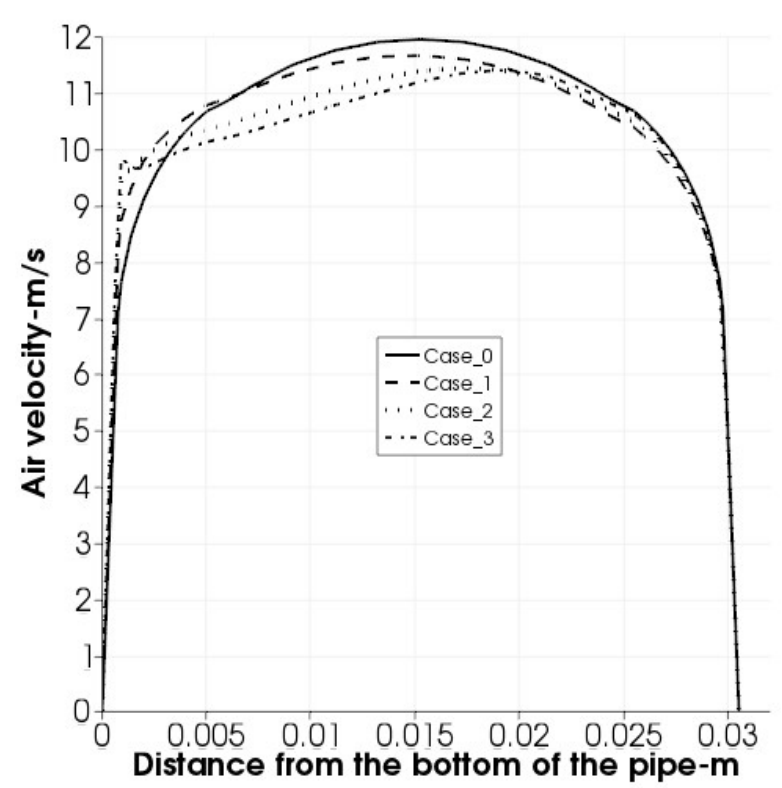

(a)

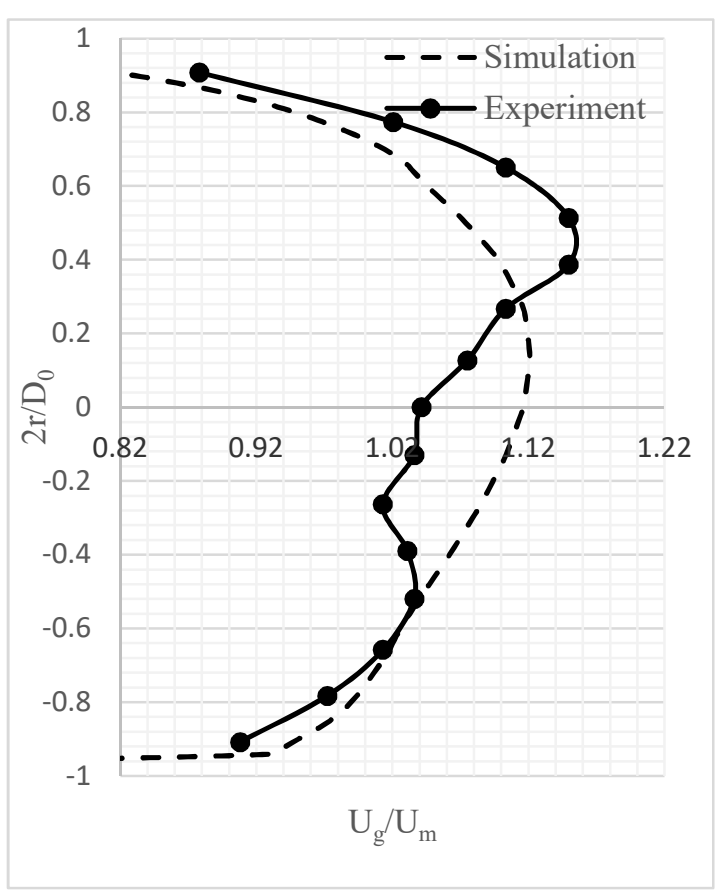

(b)

Figure 5. (a) Air velocity profiles for each case at outlet at $1.8 \mathrm{~s}$ and (b) Comparison of experimental and predicted air velocity profiles at outlet at $1.8 \mathrm{~s}$ for the Case_2.

certain set of particle-particle and particle-wall collision coefficients in the present study and some of the literature emphasizes the high sensitivity of some of these coefficients on pressure drop results (Patro and Dash, 2014a, 2014b). However, the investigation of this effect is out of the present study.

Table 2. Quasi-steady time-averaged pressure drop in fully developed region along the pipe center line.

\begin{tabular}{|l|l|l|l|}
\hline \multirow{2}{*}{ Case } & \multicolumn{2}{|l|}{ Pressure drop $(\mathrm{Pa} / \mathrm{m})$} & \multirow{2}{*}{$\begin{array}{l}\text { Error } \\
(\%)\end{array}$} \\
\cline { 2 - 3 } & Experimental & Simulation & -6.1 \\
\hline Case_0 & 52.63 & 49.4 & -11.2 \\
\hline Case_1 & 59.6 & 53.0 & -12.1 \\
\hline Case_2 & 66.2 & 58.2 & -15.1 \\
\hline Case_3 & 73.5 & 62.4 & \\
\hline
\end{tabular}

Figure 3(a) and Figure 3(b) show the air volume fraction and solids distribution, respectively, at outlet after $1.8 \mathrm{~s}$ for the case 2 . The air volume fraction is lower nearby bottom of the pipe (Figure 3 (a)) because more solids are dominated in that region (Figure 3(b)). Because of the gravity effect, the particles gradually pass off-axis and decline. Therefore, the particles tend to move to bottom side of the pipe. However, due to particle-wall collisions the particles bounce back to the core region of the pipe and are more scattered due to further particle-particle and particle-wall collisions (Figure 3(b)). Still the volume fraction of solids in bottom part of the pipe is higher compared to the upper part.
Figure 5 (a) shows the air velocity profiles at outlet at $1.8 \mathrm{~s}$ for each simulated case. Case_0 which corresponds to only air flow shows a symmetric profile. Also, it seems that the model predicts the turbulent air velocity profile quite accurately. With increase of solids loadings, the profiles pronounce the asymmetry i.e. the air velocity is getting lower in the bottom part of the pipe compared to the upper part. This can be due to more restriction for the air flow caused by high amount of solids at the bottom. However, the results in the region nearby the bottom (around $0.001 \mathrm{~m}$ in Figure 5(a)), in where the air velocities are higher for the solids loading cases (case_1, Case_2 and Case_3) than only air case (Case_0), is not as expected.

Figure 5(b) shows the comparison of experimental and simulated velocity profiles for the case 2 . The agreement between two profiles nearby bottom part of the pipe seems reasonable. However, the local minimum and maximum of the experimental profile have not been captured by the model. This can be partly due to monodispersed particles used in the simulations in contrast to poly-dispersed particles used in the experiments. Moreover, calibration of particle-wall collision parameters might be necessary for more accurate results.

\section{Conclusions}

The flow characteristics of a pneumatic conveying system are predicted using MP-PIC method in OpenFOAM. The flow is dilute and plastic pellets which are having $0.2 \mathrm{~mm}$ mean particle size and $1000 \mathrm{~kg} / \mathrm{m} 3$ 
are conveyed in $30.5 \mathrm{~mm}$ diameter pipe. The superficial air velocity was $10 \mathrm{~m} / \mathrm{s}$ and predictions are performed for 4 different solids loadings as $0,1,2$ and 3. Some of the predicted results were compared with experimental data. Predicted pressure drop results have reasonable agreement with experimental data for different loadings. The error ranges from $6-15 \%$. The solids distribution also seems physical, however no real world data is available for the comparison. Asymmetry of the gas phase velocity of the profiles due to presence of the particles have been quite well predicted by the model, however the local minimum and maximum of the experimental profile has not been captured by the present model. The discrepancies may be partly due to mono-dispersed particles used in the model in contrast to poly-dispersed particles used in the experiments. In general, it can be concluded that the used MP-PIC model gives quite reasonable predictions for dilute phase pneumatic conveying systems.

\section{Acknowledgement}

The authors would like to acknowledge the financial support provided by the Research Council of Norway under PETROMAKS II program and Det Norske oljeselskape ASA.

\section{Nomenclature}

\begin{tabular}{|c|c|}
\hline$A$ & particle accerlation $\left(\mathrm{m} / \mathrm{s}^{2}\right)$ \\
\hline$C_{D}$ & drag coefficient \\
\hline$D$ & darg funnction (s-1) \\
\hline $\mathrm{D}_{0}$ & pipe diameter $(\mathrm{m})$ \\
\hline$F$ & $\begin{array}{l}\text { rate of momentum exchange per unit volume } \\
\text { from the gas to the particle phase }(\mathrm{N} / \mathrm{m} 3)\end{array}$ \\
\hline$f$ & particle distribution function (PDF) \\
\hline$f_{D}$ & $\begin{array}{l}\text { PDF obtained by collapsing the velocity } \\
\text { dependence } \mathrm{f} \text { of to a delta function centered } \\
\text { about the local mass-averaged particle velocity }\end{array}$ \\
\hline$f_{G}$ & equilibrium distribution \\
\hline I & unit tensor \\
\hline$m$ & particle mass $(\mathrm{kg})$ \\
\hline$p$ & static pressure $(\mathrm{Pa})$ \\
\hline$P_{s}$ & pressure constant $(\mathrm{Pa})$ \\
\hline$R$ & particle radius $(\mathrm{m})$ \\
\hline $\mathrm{r}$ & vertical distance from pipe horizontal axis $(\mathrm{m})$ \\
\hline$t$ & time $(s)$ \\
\hline$u_{g}$ & gas velocity vector $(\mathrm{m} / \mathrm{s})$ \\
\hline $\mathrm{Ug}$ & axial gas velocity $(\mathrm{m} / \mathrm{s})$ \\
\hline Um & superficial air velocity at inlet $(\mathrm{m} / \mathrm{s})$ \\
\hline$v$ & particle velocity vector $(\mathrm{m} / \mathrm{s})$ \\
\hline$x$ & particle position (m) \\
\hline$\beta$ & constant \\
\hline$\gamma$ & constant \\
\hline
\end{tabular}

$\varepsilon$

$\theta \quad$ solids volume fraction

$\theta_{c p} \quad$ particle phase volume fraction at close pack

$\mu_{\text {eff }} \quad$ effective viscosity (Pa s)

$\rho_{g} \quad$ gas density $(\mathrm{kg} / \mathrm{m} 3)$

$\rho_{s} \quad$ particle density $(\mathrm{kg} / \mathrm{m} 3)$

$\tau \quad$ isotropic solids stress $(\mathrm{Pa})$

$\tau_{D} \quad$ collision damping time (s)

$\tau_{g} \quad$ gas stress tensor $(\mathrm{Pa})$

$\tau_{G} \quad$ relaxation time (s)

$\omega \quad$ constant

\section{References}

M. J. Andrews and P. J. O'Rourke. The multiphase particlein-cell (MP-PIC) method for dense particulate flows. International Journal of Multiphase Flow, 22(2): 379-402, 1996. doi:10.1016/0301-9322(95)00072-0.

H. Bilirgen and E. K. Levy. Mixing and dispersion of particle ropes in lean phase pneumatic conveying. Powder Technology, 119(2-3): 134-152, 2001. doi:10.1016/S00325910(00)00413-7.

C. Chen, J. Werther, S. Heinrich, H.-Y. Qi, and E.-U. Hartge. CPFD simulation of circulating fluidized bed risers. Powder Technology, 235: 238-247, 2013. doi:10.1016/j.powtec.2012.10.014.

K. W. Chu and A. B. Yu. Numerical simulation of complex particle-fluid flows. Powder Technology, 179(3): 104-114, 2008. doi:10.1016/j.powtec.2007.06.017.

S. E. Harris and D. G. Crighton. Solitons, solitary waves, and voidage disturbances in gas-fluidized beds. Journal of Fluid $\begin{array}{lll}\text { Mechanics, } & \text { 266: } & \text { 243-276, }\end{array}$ doi:10.1017/S0022112094000996.

M. Hidayat and A. Rasmuson. Some aspects on gas-solid flow in a U-bend: Numerical investigation. Powder Technology, 153(1): 1-13, 2005. doi:10.1016/j.powtec.2005.01.016.

N. Huber and M. Sommerfeld. Modelling and numerical calculation of dilute-phase pneumatic conveying in pipe systems. Powder Technology, 99(1): 90-101, 1998. doi:10.1016/S0032-5910(98)00065-5.

L. M. Hyder, M. S. A. Bradley, A. R. Reed, and K. Hettiaratchi. An investigation into the effect of particle size on straight-pipe pressure gradients in lean-phase conveying. Powder Technology, 112(3): 235-243, 2000. doi:10.1016/S0032-5910(00)00297-7.

S. Karimipour and T. Pugsley. Application of the particle in cell approach for the simulation of bubbling fluidized beds of Geldart A particles. Powder Technology, 220: 63-69, 2012. doi:10.1016/j.powtec.2011.09.026.

S. Laín and M. Sommerfeld. Euler/Lagrange computations of pneumatic conveying in a horizontal channel with different wall roughness. Powder Technology, 184(1): 76-88, 2008. doi:10.1016/j.powtec.2007.08.013.

L. Y. Lee, T. Yong Quek, R. Deng, M. B. Ray, and C.-H. Wang. Pneumatic transport of granular materials through a 
bend. Chemical Engineering Science, 59(21): 4637-4651, 2004. doi:10.1016/j.ces.2004.07.007.

A. Levy and D. J. Mason. The effect of a bend on the particle cross-section concentration and segregation in pneumatic conveying systems. Powder Technology, 98(2): 95-103, 1998. doi:10.1016/S0032-5910(97)03385-8.

Y. Liang, Y. Zhang, T. Li, and C. Lu. A critical validation study on CPFD model in simulating gas-solid bubbling fluidized beds. Powder Technology, 263: 121-134, 2014. doi:10.1016/j.powtec.2014.05.003.

D. J. Mason and A. Levy. A comparison of one-dimensional and three-dimensional models for the simulation of gassolids transport systems. Applied Mathematical Modelling, 22(7): 517-532, 1998. doi:10.1016/S0307-904X(98)00002$\mathrm{X}$.

D. J. Mason and J. Li. A novel experimental technique for the investigation of gas-solids flow in pipes. Powder Technology, 112(3): 203-212, 2000. doi:10.1016/S00325910(00)00294-1.

P. J. O'Rourke and D. M. Snider. Inclusion of collisional return-to-isotropy in the MP-PIC method. Chemical Engineering Science, 80: 39-54, 2012. doi:10.1016/j.ces.2012.05.047.

P. J. O'Rourke and D. M. Snider. An improved collision damping time for MP-PIC calculations of dense particle flows with applications to polydisperse sedimenting beds and colliding particle jets. Chemical Engineering Science, 65(22): 6014-6028, 2010. doi:10.1016/j.ces.2010.08.032

J. Parker, K. LaMarche, W. Chen, K. Williams, H. Stamato, and S. Thibault. CFD simulations for prediction of scaling effects in pharmaceutical fluidized bed processors at three scales. Powder Technology, 235: 115-120, 2013. doi:10.1016/j.powtec.2012.09.021.

P. Patro and S. K. Dash. Numerical Simulation for Hydrodynamic Analysis and Pressure Drop Prediction in Horizontal Gas-Solid Flows. Particulate Science and Technology, 32(1): $\quad 94-103, \quad 2014 \mathrm{a}$. doi:10.1080/02726351.2013.829543.

P. Patro and S. K. Dash. Prediction of acceleration length in turbulent gas-solid flows. Advanced Powder Technology, 25(5): 1643-1652, 2014b. doi:10.1016/j.apt.2014.05.019

S. Shah, K. Myöhänen, S. Kallio, and T. Hyppänen. CFD simulations of gas-solid flow in an industrial-scale circulating fluidized bed furnace using subgrid-scale drag models. Particuology, 18: 66-75, 2015. doi:10.1016/j.partic.2014.05.008

D. M. Snider. An Incompressible Three-Dimensional Multiphase Particle-in-Cell Model for Dense Particle Flows. Journal of Computational Physics, 170(2): 523-549, 2001. doi:10.1006/jcph.2001.6747.

Y. Tsuji and Y. Morikawa. LDV measurements of an air-solid two-phase flow in a horizontal pipe. Journal of Fluid Mechanics, $\quad 120: \quad 385-409, \quad 1982$. doi: $10.1017 / \mathrm{S} 002211208200281 \mathrm{X}$. 